\title{
Comportements malsains chez les adolescents canadiens : prévalence, tendances et corrélats
}

\author{
T. M. Gadalla, Ph. D.
}

Cet article a fait l'objet d'une évaluation par les pairs.

\section{Résumé}

Contexte : Cette étude examine les tendances temporelles associées à la prévalence de divers comportements malsains chez les adolescents âgés de 12 à 17 ans, les associations les plus courantes de comportements malsains ainsi que les corrélats socioéconomiques et sociodémographiques des comportements malsains chez les adolescents.

Méthodologie : Analyse secondaire des données recueillies auprès de 13198 répondants de l'Enquête sur la santé dans les collectivités canadiennes (ESCC) de 2000-2001 et auprès de 11050 répondants de l'ESCC de 2007-2008.

Résultats : Bien que la proportion des adolescents ayant une alimentation saine ait augmenté au cours de la période à l'étude, environ $50 \%$ d'entre eux consomment encore des quantités insuffisantes de fruits et de légumes. Dans les deux cycles, plus du tiers des adolescents de 15 à 17 ans ont déclaré consommer de l'alcool régulièrement. Il $\mathrm{y}$ avait une association statistiquement significative entre le niveau de revenu, le niveau de scolarité, le sexe ainsi que la langue parlée à la maison et le risque de comportements malsains chez les 12 à 14 ans; les mêmes associations ont été observées chez les 15 à 17 ans, sauf en ce qui concerne le niveau de revenu. Dans les deux groupes d'âge, le fait de parler une langue autre que le français ou l'anglais à la maison était associé à un faible risque de comportements malsains.

Conclusion : Nous avons observé une diminution générale des comportements malsains chez les jeunes adolescents (12-14 ans).

Mots-clés : santé des adolescents, alcool, tabagisme, alimentation saine, poids, activité physique

\section{Introduction}

Les comportements malsains à l'adolescence, comme le tabagisme, la sédentarité, une mauvaise alimentation (par exemple, consommer moins de fruits et légumes que ce qui est recommandé) et la consommation d'alcool, contribuent à l'apparition de maladies chroniques à l'âge adulte ${ }^{1,2,3}$. Les jeunes adultes qui ont déclaré avoir consommé de l'alcool pour la première fois entre 11 et 14 ans présentent un risque accru de maladie liée à l'alcool ${ }^{1}$, comme certains cancers et certaines maladies cardiaques et vasculaires ${ }^{4}$, de même qu'un risque accru d'effets néfastes sur le développement du cerveau ${ }^{4,5}$. Ces problèmes de santé chroniques ont à leur tour d'importantes répercussions négatives sur la qualité de vie et la productivitéé,

La sédentarité et la mauvaise alimentation mènent à l'embonpoint et à l'obésité, deux facteurs de risque associés à un grand nombre de complications chroniques, comme les maladies cardiovasculaires, l'hypertension artérielle, le diabète de type 2, les accidents vasculaires cérébraux, l'apnée du sommeil et certains types de cancer, de même qu'à des complications durant la grossesse ou lors d'interventions chirurgicales ${ }^{7}$. L’obésité est aussi considérée comme un facteur de risque de limitations fonctionnelles et d'une mauvaise qualité de vie sur le plan de la santé ${ }^{8,9}$.

Dans des études utilisant des échantillons nationaux d'élèves du secondaire aux États-Unis, près du quart des élèves faisaient de l'embonpoint ${ }^{10,11}$ et 13,6\% étaient obèses ${ }^{11}$. Dans un échantillon national de jeunes adultes américains âgés de 18 à 24 ans, plus des trois quarts $(78,4 \%)$ consommaient moins de cinq fruits et légumes par jour, et 43,2 \% faisaient peu ou pas d'activité physique ${ }^{12}$. Des taux similaires ont été observés chez les jeunes Canadiens : en se fondant sur des données nationales recueillies auprès d'enfants canadiens âgés de 7 à 13 ans, Tremblay et Willms ont constaté une augmentation de l'indice de masse corporelle (IMC) de l'ordre de $0,1 \mathrm{~kg} / \mathrm{m}^{2}$ par année entre 1981 et $1996^{13}$. Selon les auteurs, le taux de prévalence de l'embonpoint se situe à respectivement $28,8 \%$ chez les garçons et à 23,6\% chez les filles ${ }^{13}$.

Seo et collab. ont signalé une augmentation du taux de prévalence du tabagisme chez les élèves du secondaire, lequel est passé de 21,9 \% en 2003 à 23,0 \% en $2005^{11}$. Pisetsky et collab. ont constaté des taux similaires de tabagisme chez les adolescents $^{14}$. Environ le tiers (34,3 \%) des élèves de $7^{\mathrm{e}}$ à $12^{\mathrm{e}}$ années vivant dans les provinces canadiennes de l'Atlantique ont déclaré fumer des cigarettes ${ }^{15}$.

Rattachement de l'auteur :

Factor-Inwentash Faculty of Social Work, University of Toronto, Toronto (Ontario), Canada

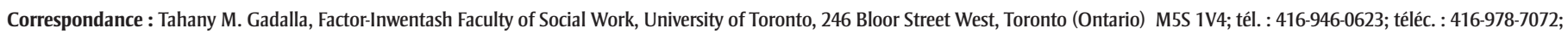
courriel : tahany.gadalla@utoronto.ca 
Seo et collab. ont également signalé l'existence d'une corrélation préjudiciable entre le tabagisme et une mauvaise alimentation ${ }^{11}$. Chez les élèves du secondaire aux États-Unis, le tabagisme était associé à l'embonpoint, et de façon plus prononcée entre 1999 et $2005^{11}$.

Pisetsky et collab. ont constaté que $22 \%$ des filles et $27,7 \%$ des garçons au secondaire consommaient périodiquement des quantités excessives d'alcool, c'est-à-dire cinq verres ou plus en une même occasion, et ce, au moins une fois par mois ${ }^{14}$. Dans un échantillon de jeunes adultes de 18 à 24 ans vivant aux États-Unis, 28,9\% ont déclaré être fumeurs, et $30,1 \%$ ont dit consommer périodiquement des quantités excessives d'alcool ${ }^{12}$. Selon une enquête nationale menée auprès de 4296 adolescents canadiens, $29 \%$ des 14 à 15 ans ont dit boire jusqu'à l'ivresse ${ }^{16}$. Plus de la moitié $(53,6 \%)$ des élèves de $7^{\text {e }}$ à $12^{\text {e }}$ années vivant dans les provinces canadiennes de l'Atlantique ont déclaré consommer de l'alcool ${ }^{15}$.

Il existe des différences dans la prévalence des comportements à risque en fonction de la situation socioéconomique, du sexe et de l'origine ethnique. Wardle et collab. ont constaté que les adolescents des quartiers défavorisés étaient plus susceptibles d'avoir essayé de fumer, d'avoir une alimentation riche en matières grasses et de faire de l'embonpoint, et ces différences se sont maintenues après ajustement en fonction de l'origine ethnique ${ }^{2}$. Par ailleurs, Tremblay et Willms ont constaté que de niveau d'activité physique et les comportements sédentaires expliquaient en partie l'association entre le statut socioéconomique et l'embonpoint/ l'obésité chez les enfants canadiens de 7 à $11 \mathrm{ans}^{17}$. Dans une étude récente menée auprès d'adolescents californiens de 12 à 17 ans, on a constaté que le taux de prévalence de l'obésité avait augmenté considérablement entre 2001 et 2007 chez les adolescents à faible revenu, mais pas chez les adolescents ayant un revenu élevé ${ }^{18}$.

Un certain nombre d'études ont montré que, chez les adolescents, les filles sont généralement plus préoccupées par leur poids que les garçons et sont plus susceptibles qu'eux de vouloir perdre du poids, en employant parfois des méthodes malsaines, comme le tabagisme, les pilules coupe-faim et les laxatifs ${ }^{10,19-21}$. Garry et collab. ont également noté que l'utilisation de pilules coupe-faim et le fait de se faire vomir / d'utiliser des laxatifs étaient fortement associés à la consommation d'alcool et au tabagisme chez les élèves des écoles intermédiaires ${ }^{20}$. Allison et collab. ont constaté que le taux de tabagisme quotidien diminue au fur et à mesure qu'augmente le niveau de scolarité, mais que cette diminution n'est pas associée au niveau de revenu ${ }^{22}$. Plus récemment, Kestila et collab. ont examiné la relation entre le contexte social durant l'enfance et l'embonpoint chez les jeunes adultes de 18 à 29 ans $^{23}$. Les chercheurs ont observé, chez les jeunes femmes, une association entre l'embonpoint et le fait d'avoir des parents peu scolarisés ou n'ayant pas d'emploi stable; cette observation ne s'est toutefois pas avérée chez les hommes. Les jeunes femmes ayant passé leur enfance en milieu rural étaient plus susceptibles d'être obèses que celles ayant passé leur enfance en milieu urbain ou semi-urbain ${ }^{23}$.

Il est essentiel de connaître le type et la fréquence des comportements malsains des adolescents, ainsi les modalités d'adoption de tels comportements, pour planifier les activités de prévention, d'intervention et de sensibilisation visant à améliorer la santé des Canadiens. Les décideurs bénéficieraient également d'une analyse des tendances temporelles (annuelles) associées aux comportements malsains et de l'identification des groupes de jeunes susceptibles de s'adonner à de tels comportements, information dont on ne dispose pas à l'heure actuelle. Cette étude vise à combler ces lacunes. Nous nous penchons plus précisément sur 1) les tendances associées à la prévalence de l'obésité ou de l'embonpoint et des comportements malsains, comme un mode de vie peu actif, une mauvaise alimentation (p. ex., consommer moins de fruits et de légumes que ce qui est recommandé pour son groupe d'âge) et la consommation d'alcool, dans un échantillon national représentatif d'adolescents canadiens de 12 à 17 ans, en 2000-2001 et en 2007-2008; 2) les associations les plus courantes de comportements malsains adoptés par les adolescents selon leur sexe et 3) les caractéristiques sociodémographiques et économiques associées aux comportements malsains chez les jeunes adolescents et les adolescents plus âgés.

\section{Méthodologie}

\section{Échantillon}

Pour effectuer cette recherche, nous avons utilisé des données recueillies dans le cadre de deux cycles de l'Enquête sur la santé dans les collectivités canadiennes (ESCC), en vertu de la Loi sur la statistique du Canada $^{24}$. Cette enquête transversale, menée tous les deux ans, utilise un échantillon aléatoire à grappes stratifié à plusieurs degrés pour lequel les logements constituent l'unité d'échantillonnage final. L'échantillon a été stratifié par province/territoire et par région urbaine ou rurale dans chaque province/territoire. L'échantillonnage a été réalisé de manière à être représentatif de $98 \%$ de la population canadienne âgée de 12 ans ou plus vivant dans des logements privés dans les dix provinces et les trois territoires du pays. Nous avons choisi au hasard environ la moitié des répondants de chacun des cycles de l'enquête et nous les avons convoqués à une rencontre au cours de laquelle nous les avons sondés en utilisant une méthode d'entrevue en face-à-face assistée par ordinateur; l'autre moitié des répondants a été sondée par téléphone au moyen d'une méthode d'entrevue téléphonique assistée par ordinateur ${ }^{24,25}$. Une lettre explicative a été envoyée aux répondants choisis pour les informer des lois sur la confidentialité qui régissent la communication et/ou la publication des données recueillies et pour les informer du fait qu'ils sont tout à fait libres d'accepter ou de refuser de participer à l'étude. Les intervieweurs ont obtenu l'autorisation verbale des parents ou des tuteurs légaux des jeunes âgés de 12 à 15 ans et ont expliqué la raison pour laquelle ils recueillaient ces données, les sujets qui seraient abordés et la nécessité de respecter la vie privée et le droit à la confidentialité des enfants. Les cas où il n'était pas possible de discuter seul à seul avec un jeune, que ce soit en personne ou au téléphone, ont été codés comme des refus. On trouvera de plus amples renseignements sur la conception de l'enquête 
dans d'autres publications ${ }^{24,25}$. L'échantillon compte 13198 répondants de 12 à 17 ans ayant participé à l'enquête de 2000-2001 et 11050 répondants ayant participé à celle de 2007-2008.

\section{Mesures}

La consommation d'alcool a été mesurée à l'aide de deux variables : la fréquence de la consommation d'alcool et la consommation périodique de quantités excessives d'alcool. La fréquence de la consommation d'alcool a été déterminée à partir des habitudes des répondants au cours des 12 mois précédant l'enquête. Nous avons établi les catégories suivantes : consommation régulière d'alcool (au moins une fois par mois), consommation occasionnelle d'alcool (moins d'une fois par mois) et aucune consommation d'alcool. Nous avons également défini la consommation périodique de quantités excessives d'alcool comme la consommation de cinq verres d'alcool ou plus en une seule occasion, au moins une fois par mois.

Le tabagisme a été mesuré à l'aide d'une seule variable : la fréquence. Les répondants ont dû répondre à la question suivante : " À l'heure actuelle, fumez-vous des cigarettes tous les jours, à l'occasion ou pas du tout? », et nous nous sommes fondés sur leur réponse pour les classer dans les catégories "fumeur quotidien ", " fumeur occasionnel » et « non-fumeur ».

La classification en fonction de l'IMC était fondée sur les seuils spécifiques en fonction de l'âge et du sexe définis par Cole et collab. pour les jeunes de 12 à 18 ans $^{26}$, seuils qui ont été déterminés à partir de données recueillies dans diverses régions du monde, à savoir au Brésil, en GrandeBretagne, à Hong Kong, aux Pays-Bas, à Singapour et aux États-Unis ${ }^{26}$. Les auteurs ont utilisé la taille et le poids de plus de 192000 personnes pour calculer des seuils spécifiques en fonction de l'âge et du sexe et définir ainsi des catégories d'IMC s'appliquant aux 12 à 18 ans. Les seuils ont été déterminés pour chaque sexe et chaque âge entre 12 ans et 18 ans; chez les garçons, ces seuils variaient entre $21,22 \mathrm{~kg} / \mathrm{m}^{2}$ et $30,0 \mathrm{~kg} / \mathrm{m}^{2}$, et chez les filles, entre $21,68 \mathrm{~kg} / \mathrm{m}^{2}$ et $30,0 \mathrm{~kg} / \mathrm{m}^{2}$. Cette variable permet de classer les adolescents (à l'exception des filles de 15 à 17 ans qui étaient enceintes ou qui n'avaient pas indiqué si elles l'étaient ou non) dans les catégories "obésité », « embonpoint » et « ni l'un ni l'autre ».

Nous avons également utilisé d'autres variables associées à la santé dans notre analyse, notamment la consommation quotidienne de fruits et de légumes (moins de cinq portions et cinq portions ou plus) comme indicateur d'une mauvaise alimentation, et l'autoperception de son état de santé général (excellent/très bon, bon et passable/médiocre). Les données sur l'autoperception du degré de stress ressenti ont été recueillies uniquement auprès des répondants de 15 ans ou plus, lesquels ont dû répondre à la question suivante : "En ce qui concerne le stress, diriez-vous que vos journées sont, de façon générale, pas stressantes du tout, pas très stressantes, un peu stressantes, plutôt stressantes ou extrêmement stressantes?»

L'activité physique a été mesurée à l'aide de deux variables : le degré d'activité physique et le temps consacré à des activités sédentaires. La catégorisation du degré d'activité physique est fondée sur la dépense énergétique (DE) quotidienne associée au transport et aux loisirs : " actif » pour une DE supérieure à 3,0 kcal/kg/jour, « modérément actif » pour une DE comprise entre 1,5 et 3,0 kcal $/ \mathrm{kg} /$ jour et « sédentaire » pour une DE inférieure à 1,5 kcal/kg/jour. La dépense énergétique des répondants a été calculée à l'aide de la fréquence et de la durée des séances pour chaque type d'activité physique et du coût énergétique (coefficient MET) associé à ces activités. Par exemple, pour effectuer une activité associée à un coût énergétique de 4 MET, il faut fournir quatre fois plus d'énergie que lorsque le corps est au repos. Nous avons calculé la dépense énergétique (coefficient MET) associée à une séance de 15 minutes pour chaque activité, et nous avons multiplié le résultat par le nombre de séances pour obtenir la dépense énergétique totale correspondant à chaque activité. Nous n'avons pas demandé aux répondants de préciser avec quelle intensité ils pratiquaient leurs activités; par conséquent, les coefficients MET utilisés dans la présente étude correspondent à un degré d'intensité faible. Nous avons décidé de procéder ainsi parce que les gens ont tendance à surestimer l'intensité, la fréquence et la durée de leurs activités ${ }^{24}$.

Nous avons également estimé le nombre total d'heures consacrées à des activités sédentaires au cours d'une semaine type dans les 3 mois ayant précédé l'enquête. Les activités sédentaires englobaient notamment le fait d'utiliser un ordinateur (en particulier pour jouer et pour naviguer sur Internet), de jouer à des jeux vidéo, de regarder la télévision ou des vidéos, et de lire. Le temps passé à l'école ou au travail n’a pas été pris en considération. Nous avons ensuite établi quatre catégories pour ce qui concerne les activités sédentaires des répondants : moins de 15 heures/ semaine, de 15 à 29 heures/semaine, de 30 à 44 heures/semaine et 45 heures/ semaine ou plus.

Nous avons utilisé pour cette étude les caractéristiques sociodémographiques suivantes : le sexe, le groupe d'âge (12-14 ans ou 15-17 ans), la langue parlée à la maison (l'anglais/le français ou une autre langue), le lieu de naissance (le Canada ou un autre pays), le niveau de scolarité le plus élevé ayant été atteint au sein du ménage (études secondaires non terminées, diplôme d'études secondaires, études postsecondaires non terminées, diplôme d'études postsecondaires) et le niveau de revenu. Il faut cependant préciser que les données sur le niveau de revenu n'ont pas été présentées de la même façon dans les deux cycles de l'ESCC : il y avait quatre catégories de niveau de revenu dans le cycle de 2000-2001, et seulement trois dans celui de 2007-2008 ${ }^{24}$. Par conséquent, nous n'avons pas pu effectuer une comparaison directe de cette variable.

\section{Analyse des données}

Nous avons calculé et comparé, pour chaque groupe d'âge, les taux selon lesquels les participants adoptaient des comportements associés à un risque sur le plan de la santé pour 2000-2001 et 2007-2008, et nous avons utilisé des tests du chi carré $\left(\chi^{2}\right)$ pour comparer le taux de prévalence des comportements malsains dans les deux 
cycles de l'ESCC. Nous avons également utilisé des tests du $\chi^{2}$ pour analyser les relations bivariées entre les comportements malsains et divers attributs sociodémographiques et économiques.

Dans le cas des comportements malsains pour lesquels il existe plus de deux catégories, nous avons consigné les résultats au moyen de variables oui/non. Par exemple, nous avons attribué le code oui à la catégorie " sédentaire » et le code non aux catégories " actif » et " modérément actif », le code oui aux catégories " fumeur quotidien » et " fumeur occasionnel » et le code non à la catégorie " non-fumeur », le code oui aux catégories « consomme régulièrement de l'alcool » et " consomme occasionnellement de l'alcool» et le code non à la catégorie " ne consomme jamais d'alcool ». Nous avons ensuite regroupé les données pour mettre en lumière les principales associations de comportements malsains adoptés à l'adolescence par les garçons et par les filles (séparément).

Nous avons utilisé des modèles de régression logistique pour examiner les effets des attributs sociodémographiques et économiques associés à l'adoption de comportements malsains chez les jeunes adolescents et chez les adolescents plus âgés. Nous avons inclus dans l'analyse multivariée uniquement les variables pour lesquelles nous avions observé une association statistiquement significative avec l'adoption de comportements malsains dans les analyses bivariées. Les poids d'échantillonnage ont été remis à l'échelle et utilisés dans toutes les analyses. Le fait de remettre à l'échelle les poids d'échantillonnage de façon à obtenir une moyenne de 1 présente deux avantages : primo, cela permet de tenir compte de l'inégalité des probabilités associées à la sélection des répondants et d'ajuster les résultats en fonction de la composition démographique de la population canadienne, de façon qu'ils soient représentatifs de la population canadienne et non uniquement de l'échantillon; secundo, cela permet de conserver la même taille d'échantillon, ce qui empêche une éventuelle augmentation de la taille d'échantillon à l'étape de la vérification des hypothèses ${ }^{24,25}$.

\section{Résultats}

Le tableau 1 présente les statistiques descriptives associées aux variables utilisées dans notre analyse. Le changement le plus important que nous ayons constaté au cours de la période à l'étude était une augmentation de neuf points de pourcentage dans la proportion d'adolescents consommant cinq fruits et légumes ou plus par jour (12 à 14 ans : $\chi^{2}=729,33, p<0,001 ; 15$ à 17 ans : $\left.\chi^{2}=65,90, p<0,001\right)$. Nous avons également observé une diminution marquée du taux de prévalence du tabagisme dans les deux groupes d'âge (12 à 14 ans : $\chi^{2}=96,79, p<0,001$; 15 à 17 ans : $\left.\chi^{2}=120,53, p<0,001\right)$.

Bien que les données aient révélé une légère amélioration quant à la proportion des adolescents qui sont physiquement actifs, le nombre d'heures consacrées à des activités sédentaires a crû encore davantage : la proportion des adolescents consacrant plus de 45 heures/semaine à des activités sédentaires est passée de $6,1 \%$ à $8,3 \%$ chez les plus jeunes (12 à 14 ans : $\chi^{2}=42,69, p<0,001$ ) et de $3,8 \%$ à $9,0 \%$ chez les plus âgés (15 à 17 ans : $\chi^{2}=170,00, p<0,001$ ). Il y avait également une amélioration statistiquement significative de la distribution de l'IMC chez les jeunes adolescents (12 à 14 ans : $\chi^{2}=23,43$, $p<0,001$ ), mais pas chez les plus âgés. De façon analogue, la prévalence des cas de consommation excessive d'alcool s'est améliorée de façon notable chez les plus jeunes (12 à 14 ans : $\chi^{2}=13,30$, $p<0,001$ ), mais pas chez les plus âgés.

Le tableau 2 présente le nombre de comportements malsains adoptés par les adolescents et la distribution de ces comportements en fonction de l'âge et du sexe. On peut constater une augmentation considérable de la proportion de jeunes adolescents (12 à 14 ans) n'ayant pas adopté de comportement malsain entre 2000-2001 et 2007-2008 (de 25,2 \% à $36,4 \%$ chez les garçons et de 26,7 \% à 38,5\% chez les filles). Chez les adolescents plus âgés (15 à 17 ans), cette augmentation est plus modeste (de $15,7 \%$ à 20,8 \% chez les garçons et de $18,3 \%$ à 20,3\% chez les filles).
Chez les adolescents des deux sexes qui présentaient un comportement malsain, le comportement le plus répandu était la consommation de moins de cinq portions de fruits et de légumes $(57,3 \%$ chez les garçons et $47,9 \%$ chez les filles). On trouvera dans le tableau 3 d'autres exemples de comportements malsains en fonction de leur fréquence et du sexe. Chez les garçons, l'embonpoint ou l'obésité $(15,6 \%)$ et la sédentarité (15,0 \%) arrivaient au deuxième rang des attributs associés à un risque pour la santé et étaient suivis par la consommation régulière d'alcool $(10,5 \%)$ et le tabagisme quotidien (1,3\%). Chez les filles, 30,0\% étaient sédentaires, 11,6\% consommaient régulièrement de l'alcool, 9,0 \% faisaient de l'embonpoint ou étaient obèses et 1,6\% fumaient quotidiennement.

L'association la plus courante chez les adolescents présentant deux comportements malsains était la consommation de quantités insuffisantes de fruits et de légumes et la sédentarité $(35,3 \%$ chez les garçons et $51,1 \%$ chez les filles) (tableau 3). La deuxième association la plus courante chez les garçons était la consommation de quantités insuffisantes de fruits et de légumes et l'embonpoint ou l'obésité $(27,8 \%)$, tandis que chez les filles, il s'agissait de la consommation de quantités insuffisantes de fruits et de légumes et la consommation régulière d'alcool (13,7 \%). On a constaté que $8,2 \%$ des garçons et $5,7 \%$ des filles présentant une association de deux comportements malsains consommaient régulièrement de l'alcool et buvaient périodiquement des quantités excessives d'alcool. Seulement 1,8 \% des garçons et 1,4\% des filles consommaient régulièrement de l'alcool et fumaient quotidiennement (tableau 3).

Selon les tests du $\chi^{2}$ bivariés, le sexe, le niveau de revenu, le niveau de scolarité et la langue parlée à la maison étaient associés à l'adoption de comportements malsains par les jeunes adolescents (12 à 14 ans), mais pas le lieu de naissance. Chez les plus âgés (15 à 17 ans), seuls le sexe, le niveau de scolarité et la langue parlée à la maison étaient associés de manière statistiquement significative à l'adoption de comportements malsains; le degré de stress perçu par les 
TABLEAU 1

Caractéristiques et statistiques descriptives associées aux répondants adolescents (âgés de 12 à 17 ans) dans les échantillons de l’Enquête sur la santé dans les collectivités canadiennes de 2000-2001 et de 2007-2008

\begin{tabular}{|c|c|c|c|c|}
\hline & \multicolumn{4}{|c|}{ Répondants de l'ESCC, n (\%) } \\
\hline & \multicolumn{2}{|c|}{12 à 14 ans } & \multicolumn{2}{|c|}{15 à 17 ans } \\
\hline & $\begin{array}{l}2000-2001 \\
(n=6251)\end{array}$ & $\begin{array}{l}2007-2008 \\
(n=5574)\end{array}$ & $\begin{array}{l}2000-2001 \\
(n=6947)\end{array}$ & $\begin{array}{r}2007-2008 \\
(n=5476)\end{array}$ \\
\hline Filles & $2993(47,9)$ & $2664(48,7)$ & $3459(49,8)$ & $2705(49,4)$ \\
\hline \multicolumn{5}{|l|}{ Revenu $^{a}$} \\
\hline Élevé 30 \% & - & $1684(37,0)$ & - & $1267(32,1)$ \\
\hline Faible $30 \%$ & - & $1034(22,6)$ & - & $943(23,0)$ \\
\hline \multicolumn{5}{|l|}{ Langue parlée à la maison } \\
\hline Anglais/français & - & $5057(90,7)$ & - & $2914(89,7)$ \\
\hline Autre & - & $517(9,3)$ & - & $561(10,3)$ \\
\hline Études secondaires non terminées & $508(8,3)$ & $188(4,3)$ & $533(7,9)$ & $186(4,2)$ \\
\hline Diplôme d'études secondaires & $898(14,7)$ & $478(10,9)$ & $986(14,6)$ & $576(12,9)$ \\
\hline Études postsecondaires non terminées & $449(7,3)$ & $248(5,6)$ & $621(9,2)$ & $305(6,8)$ \\
\hline Diplôme d'études postsecondaires & $4264(69,7)$ & $3487(79,2)$ & $4627(68,4)$ & $3410(76,2)$ \\
\hline \multicolumn{5}{|l|}{ État de santé perçu } \\
\hline Excellent/très bon & $4546(72,7)$ & $3902(70,1)$ & $4877(70,2)$ & $3749(68,5)$ \\
\hline Bon & $1461(23,4)$ & $1478(26,5)$ & $1690(24,3)$ & $1438(26,3)$ \\
\hline Passable/médiocre & $242(3,9)$ & $191(3,4)$ & $378(5,4)$ & $289(5,3)$ \\
\hline \multicolumn{5}{|l|}{ Niveau de stress perçu ${ }^{b}$} \\
\hline Embonpoint $^{\mathrm{d}}$ & $1011(17,0)$ & $643(14,1)$ & $981(14,4)$ & $758(15,1)$ \\
\hline Ni l'un ni l'autre & $4636(77,8)$ & $3724(81,7)$ & $5528(81,2)$ & $4028(80,3)$ \\
\hline \multicolumn{5}{|c|}{ Consommation quotidienne de fruits et de légumes, portions } \\
\hline Moins de 5 & $3591(58,5)^{* *}$ & $2486(50,1)$ & $4050(59,1)^{* *}$ & $2665(51,6)$ \\
\hline 5 et plus & $2547(41,5)$ & $4608(49,9)$ & $2808(40,9)$ & $2497(49,4)$ \\
\hline \multicolumn{5}{|l|}{ Activité physique } \\
\hline Actif & $2595(49,4)^{*}$ & $2724(51,7)$ & $2699(44,0)^{* *}$ & $2583(48,5)$ \\
\hline Modérément actif & $1347(25,6)$ & $1202(22,8)$ & $1461(23,8)$ & $1150(21,6)$ \\
\hline Sédentaire & $1310(24,9)$ & $1346(25,5)$ & $1976(32,2)$ & $1597(30,0)$ \\
\hline \multicolumn{5}{|l|}{ Activités sédentaires, heures/semaine } \\
\hline Moins de 15 & $973(30,1)^{* *}$ & $1345(26,0)$ & $1404(35,7)^{* *}$ & $1414(26,7)$ \\
\hline 15 à 29 & $1481(45,8)$ & $2256(43,6)$ & $1761(44,8)$ & $2358(44,6)$ \\
\hline 30 à 44 & $583(18,0)$ & $1145(22,1)$ & $615(15,6)$ & $1045(19,8)$ \\
\hline 45 et plus & $198(6,1)$ & $427(8,3)$ & $149(3,8)$ & $474(9,0)$ \\
\hline
\end{tabular}


TABLEAU 1 (Suite)

Caractéristiques et statistiques descriptives associées aux répondants adolescents (âgés de 12 à 17 ans) dans les échantillons de l'Enquête sur la santé dans les collectivités canadiennes de 2000-2001 et de 2007-2008

\begin{tabular}{|c|c|c|c|c|}
\hline & \multicolumn{4}{|c|}{ Répondants de l’ESCC, n (\%) } \\
\hline & \multicolumn{2}{|c|}{12 à 14 ans } & \multicolumn{2}{|c|}{15 à 17 ans } \\
\hline & $\begin{array}{l}2000-2001 \\
(n=6251)\end{array}$ & $\begin{array}{l}2007-2008 \\
(n=5574)\end{array}$ & $\begin{array}{l}2000-2001 \\
(n=6947)\end{array}$ & $\begin{array}{l}2007-2008 \\
(n=5476)\end{array}$ \\
\hline \multicolumn{5}{|l|}{ Tabagisme } \\
\hline Quotidien & $185(3.0)^{* *}$ & $51(0.9)$ & $1005(14.5)^{* *}$ & $450(8.2)$ \\
\hline Occasionnel & $190(3.0)$ & $82(1.5)$ & $435(6.3)$ & 307 (5.6) \\
\hline \multicolumn{5}{|l|}{ Consommation d'alcool } \\
\hline Régulièrement & $335(5.4)^{* *}$ & 219 (3.9) & $2288(33.2)^{*}$ & $1859(34.3)$ \\
\hline Occasionnellement & $1069(17.2)$ & $747(13.5)$ & $1987(28.9)$ & $1410(26.0)$ \\
\hline Jamais & 3809 (77.4) & $4581(82.6)$ & 2609 (37.9) & $2155(39.7)$ \\
\hline
\end{tabular}

Abréviations : ESCC, Enquête sur la santé dans les collectivités canadiennes; IMC, indice de masse corporelle.

${ }^{a}$ Les catégories de niveau de revenu n'étaient pas identiques dans l'ESCC de 2000-2001 et celle de 2007-2008.

${ }^{b}$ Question posée uniquement aux répondants de 15 ans et plus en 2007-2008.

${ }^{\mathrm{c}}$ IMC de $26,02 \mathrm{~kg} / \mathrm{m}^{2}$ chez les garçons âgés de 12 à 14 ans, de $>30,0 \mathrm{~kg} / \mathrm{m}^{2}$ chez les garçons âgés de 15 à 17 ans, de $26,67 \mathrm{~kg} / \mathrm{m}^{2}$ chez les filles âgées de 12 à 14 ans, et de $>30,00 \mathrm{~kg} / \mathrm{m}^{2}$ chez les filles âgées de 15 à 17 ans.

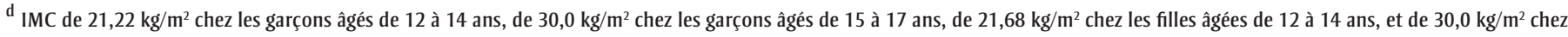
les filles âgées de 15 à 17 ans.

* $p<0,01$ pour la différence relative à la prévalence au cours de la période à l'étude (tests du $\chi^{2}$ ).

${ }^{* *} p<0,001$ pour la différence relative à la prévalence au cours de la période à l'étude (tests du $\chi^{2}$ ).

TABLEAU 2

Répondants adolescents (âgés de 12 à 17 ans) sondés dans le cadre de l'Enquête sur la santé dans les collectivités canadiennes ayant adopté des comportements malsains, par groupe d'âge et par sexe, en 2000-2001 et en 2007-2008

\begin{tabular}{|c|c|c|c|c|c|c|c|}
\hline \multirow[t]{2}{*}{ Groupe d'âge } & \multirow[t]{2}{*}{ Répondants, n (\%) } & \multicolumn{6}{|c|}{ Nombre de comportements malsains } \\
\hline & & $\mathbf{0}$ & 1 & 2 & 3 & 4 et plus & Total \\
\hline \multirow[t]{3}{*}{12 à 14 ans } & $2000 / 2001^{*}$ & & & & & & \\
\hline & Filles & $800(26,7)$ & $1323(44,2)$ & $710(23,7)$ & $129(4,3)$ & $32(1,1)$ & 2993 \\
\hline & $2007-2008$ & & & & & & \\
\hline \multirow[t]{4}{*}{15 à 17 ans } & $2000-2001^{*}$ & & & & & & \\
\hline & Garçons & $546(15,7)$ & $1139(32,7)$ & $999(28,6)$ & $498(14,3)$ & $307(8,8)$ & 3488 \\
\hline & Filles & $631(18,3)$ & $1152(33,3)$ & $972(28,1)$ & $416(12,0)$ & $288(8,4)$ & 3459 \\
\hline & $2007-2008^{* *}$ & & & & & & \\
\hline
\end{tabular}

Abréviation : ESCC, Enquête sur la santé dans les collectivités canadiennes.

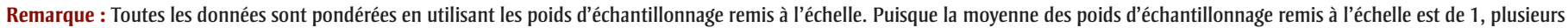
données seraient des fractions. Par conséquent, les totaux obtenus dans les différentes analyses peuvent ne pas être tout à fait égaux en raison de l'approximation.

${ }^{*} p<0,01$ pour la différence entre les garçons et les filles (tests du $\chi^{2}$ ).

${ }^{* *} p<0,001$ pour la différence entre les garçons et les filles (tests du $\chi^{2}$ ). 
répondants, le lieu de naissance et le revenu n'étaient pas liés à l'adoption de tels comportements.

Selon l'analyse de régression logistique, le niveau de scolarité, le sexe et la langue parlée à la maison étaient associés de façon statistiquement significative à la probabilité que les adolescents adoptent au moins un comportement malsain (tableau 4). Ces probabilités étaient légèrement plus élevées chez les garçons de 12 à 14 ans que chez les filles du même âge (rapport de cotes $[\mathrm{RC}]=1,18$, intervalle de confiance [IC] à $95 \%=1,03$ à 1,34), mais elles étaient plus faibles chez les garçons de 15 à 17 ans que chez les filles du même âge ( $\mathrm{RC}=0,83$, IC à $95 \%=0,70$ à 0,97$)$. Les répondants qui parlaient une autre langue que l'anglais ou le français à la maison étaient moins susceptibles d'adopter un comportement malsain (12 à 14 ans : $\mathrm{RC}=0,66$, IC à $95 \%=0,51$ à 0,85 ; 15 à 17 ans : $\mathrm{RC}=0,60$, IC à $95 \%=$ $0,46$ à 0,80$)$. Dans les ménages où le niveau de scolarité le plus élevé était un diplôme d'études secondaires, les adolescents présentaient un risque presque deux fois plus élevé d'adopter un comportement malsain que dans les ménages où le niveau de scolarité le plus élevé était un diplôme d'études postsecondaires (12 à 14 ans : RC = 1,93, IC à $95 \%=1,51$ à 2,46; 15 à 17 ans : $\mathrm{RC}=1,46$, IC à $95 \%=1,11$ à 1,92).

\section{Analyse}

Dans cette étude, nous avons examiné la prévalence du tabagisme, de l'obésité et de l'embonpoint, de la sédentarité, d'une mauvaise alimentation et de la consommation d'alcool dans un échantillon national représentatif d'adolescents canadiens pour 2000-2001 et pour 20072008. Cette étude a également distingué les tendances relatives à l'adoption de ces comportements chez les plus jeunes (12-14 ans) et chez les plus âgés (15-17 ans), de même que chez les garçons et chez les filles. Les types de comportements malsains adoptés par les adolescents, les associations les plus courantes de ces comportements ainsi que les corrélats sociodémographiques et économiques associés à l'adoption de ces comportements ont également été analysés.

L'une des limitations de la présente étude est le fait que les mesures utilisées étaient toutes fondées sur des données autodéclarées, lesquelles sont sujettes à un biais.
Bien que chez les jeunes adolescents les garçons aient été légèrement plus susceptibles d'adopter des comportements malsains que les filles, chez les plus vieux, les garçons étaient légèrement moins susceptibles que les filles d'adopter de tels comportements, ce qui est plutôt étonnant et mérite d'être approfondi. Cette observation souligne l'importance d'examiner les comportements des adolescents séparément, selon leur sexe et leur âge.

Malgré l'augmentation de la proportion d'adolescents consommant des quantités suffisantes de fruits et de légumes quotidiennement, environ la moitié d'entre eux en consommaient encore une quantité inférieure aux recommandations en 20072008. Toutefois, cette proportion est beaucoup moins élevée que les $78 \%$ de jeunes aux États-Unis qui consomment une quantité de fruits et de légumes inférieure aux recommandations ${ }^{12}$. Bien que le taux d'obésité et d'embonpoint ait diminué de $22,2 \%$ à $18,3 \%$ chez les adolescents de 12 à 14 ans au cours de la période à l'étude, ce taux est demeuré pratiquement inchangé, à 19,7 \%, chez les 15 à 17 ans. Si ces taux sont inférieurs à ceux observés dans les années $1990^{13}$, il n'en demeure pas moins qu'ils sont toujours loin d'être idéaux et que les décideurs

TABLEAU 3

Types de comportements malsains adoptés par les répondants adolescents sondés dans le cadre de l’Enquête sur la santé dans les collectivités canadiennes, par sexe, pour 2007-2008

\begin{tabular}{|c|c|c|c|}
\hline \multicolumn{2}{|r|}{ Nombre et type/association de comportements malsains } & \multicolumn{2}{|c|}{ Répondants de l’ESCC, n (\%) } \\
\hline & & $\begin{array}{c}\text { Garçons } \\
(n=2035)\end{array}$ & $\begin{array}{c}\text { Filles } \\
(n=1986)\end{array}$ \\
\hline \multirow[t]{6}{*}{ Un } & Moins de 5 portions de fruits et légumes par jour & $1171(57,3)$ & $951(47,9)$ \\
\hline & Embonpoint/obésité & $318(15,6)$ & $178(9,0)$ \\
\hline & Sédentarité & $305(15,0)$ & $595(30,0)$ \\
\hline & Consommation régulière d'alcool & $214(10,5)$ & $231(11,6)$ \\
\hline & Tabagisme quotidien & $26(1,3)$ & $31(1,6)$ \\
\hline & & $\begin{array}{c}\text { Garçons } \\
(n=1347)\end{array}$ & $\begin{array}{c}\text { Filles } \\
(n=1337)\end{array}$ \\
\hline \multirow[t]{8}{*}{ Deux } & Moins de 5 portions de fruits et légumes + sédentarité & $467(34,7)$ & $683(51,1)$ \\
\hline & Moins de 5 portions de fruits et légumes + embonpoint/obésité & $374(27,8)$ & $156(11,7)$ \\
\hline & Moins de 5 portions de fruits et légumes + cons. régulière d'alcool & $176(13,1)$ & $183(13,7)$ \\
\hline & Cons. régulière d'alcool + cons. de quantités excessives d'alcool & $111(8,2)$ & $76(5,7)$ \\
\hline & Cons. régulière d'alcool + embonpoint/obésité & $73(5,4)$ & $37(2,8)$ \\
\hline & Cons. régulière d'alcool + sédentarité & $34(2,5)$ & $76(5,7)$ \\
\hline & Cons. régulière d'alcool + tabagisme quotidien & $24(1,8)$ & $18(1,4)$ \\
\hline & Moins de 5 portions de fruits et légumes + tabagisme quotidien & $10(0,7)$ & $26(1,9)$ \\
\hline
\end{tabular}

Abréviations : Cons., consommation; ESCC, Enquête sur la santé dans les collectivités canadiennes. 
TABLEAU 4

Résultats de l'analyse de régression logistique des corrélats sociodémographiques et économiques associés à l'adoption de comportements malsains par les adolescents de 12 à 17 ans au Canada, en 2007-2008

\begin{tabular}{|c|c|c|c|}
\hline $\begin{array}{l}\text { Groupe } \\
\text { d'âge }\end{array}$ & Variable & $\begin{array}{c}\text { RC } \\
\text { (IC à } 95 \% \text { ) }\end{array}$ & $p$ \\
\hline \multirow[t]{15}{*}{12 à 14 ans } & Distribution du revenu du ménage & & \\
\hline & Faible (30 \%) & $1,20(1,00-1,44)$ & ,053 \\
\hline & Moyen (40 \%) & $1,23(1,03-1,45)$ & ,017 \\
\hline & Élevé (30%) & 1,00 (réf.) & - \\
\hline & Niveau de scolarité au sein du ménage & & \\
\hline & Études secondaires non terminées & $1,47(1,04-2,08)$ & 027 \\
\hline & Diplôme d'études secondaires & $1,93(1,51-2,46)$ & $<, 001$ \\
\hline & Études postsecondaires non terminées & $1,27(0,96-1,70)$ & , 100 \\
\hline & Diplôme d'études secondaires & 1,00 (réf.) & - \\
\hline & Langue parlée à la maison & & \\
\hline & Anglais/français & 1,00 (réf.) & - \\
\hline & Autre & $0,66(0,51-0,85)$ & $<, 001$ \\
\hline & Sexe & & \\
\hline & Masculin & $1,18(1,03-1,34)$ & ,017 \\
\hline & Féminin & 1,00 (réf.) & - \\
\hline \multirow[t]{11}{*}{15 à 17 ans } & Niveau de scolarité au sein du ménage & & \\
\hline & Études secondaires non terminées & $1,53(0,94-2,49)$ & ,087 \\
\hline & Diplôme d'études secondaires & $1,46(1,11-1,92)$ & ,006 \\
\hline & Études postsecondaires non terminées & $1,62(1,10-2,40)$ & ,015 \\
\hline & Diplôme d'études secondaires & 1,00 (réf.) & - \\
\hline & Langue parlée à la maison & & \\
\hline & Anglais/français & 1,00 (réf.) & - \\
\hline & Autre & $0,60(0,46-0,80)$ & $<, 001$ \\
\hline & Sexe & & \\
\hline & Masculin & $0,83(0,70-97)$ & ,022 \\
\hline & Féminin & 1,00 (réf.) & - \\
\hline
\end{tabular}

Abréviations : IC, intervalle de confiance; RC, rapport de cotes; réf., référence.

politiques et les personnes qui font la promotion d'un mode de vie sain doivent y porter attention.

Au cours de la période à l'étude, environ le tiers des 15 à 17 ans ont dit avoir consommé de l'alcool régulièrement au cours de l'année précédente, une proportion semblable à ce qui a été indiqué dans d'autres études ${ }^{12}$.

Selon la régression logistique, la langue parlée à la maison et le niveau de scolarité des parents sont les principaux corrélats démographiques associés aux comportements malsains chez les adolescents. Les adolescents qui parlent une langue autre que l'anglais ou le français à la maison étaient beaucoup moins susceptibles d'adopter des comportements malsains. Nous avons également observé que, moins le niveau de scolarité des parents était élevé, plus la probabilité que les jeunes adolescents comme les plus âgés adoptent un comportement malsain était élevée, observation corroborée par d'autres travaux de recherche ${ }^{23}$. Par ailleurs, nous avons constaté qu'un faible niveau de revenu était associé à un risque accru de comportements malsains chez les jeunes adolescents, mais non chez les plus âgés.

\section{Conclusion}

Cette étude repose sur une analyse de données secondaires recueillies au moyen de deux échantillons nationaux représentatifs d'adolescents de 12 à 17 ans, à savoir un échantillon associé à l'ESCC de 2000-2001 et un échantillon associé à l’ESCC de 2007-2008. L'étude a révélé une baisse générale des comportements malsains chez les jeunes adolescents (12-14 ans). Il faudrait cibler davantage les adolescents les plus âgés au moyen de programmes de sensibilisation et d'éducation en matière de santé, en mettant particulièrement l'accent sur la lutte contre les effets néfastes d'une mauvaise alimentation, de la sédentarité et de la consommation d'alcool.

\section{Références}

1. DeWit DJ, Adlaf EM, Offord DR, Ogborne AC. Age at first alcohol use: a risk factor for the development of alcohol disorders. Am J Psychiatry. 2000;157:745-50.

2. Wardle J, Jarvis MJ, Steggles N, Sutton S, Williamson S, Farrimond $\mathrm{H}$, et collab. Socioeconomic disparities in cancer-risk behaviors in adolescence: Baseline results from the Health and Behaviour in Teenagers Study (HABITS). Prev Med. 2003;36(6):721-30.

3. Sturm R. The effects of obesity, smoking, and drinking on medical problems and costs. Health Aff. 2002;21(2):245-53.

4. Windle M, Windle RC. Alcohol and other substance use and abuse. Dans : Adams GR, Berzonsky MD (dir.). Blackwell handbook of adolescence. Malden (MA) : Blackwell; 2003. p. 450-69.

5. Lemstra $M$, Bennett NR, Neudorf C, Kunst A, Nannapaneni U, Warren LM, et collab. A meta-analysis of marijuana and alcohol use by socio-economic status in adolescents aged 10-15 years. Can J Public Health. 2008;99(3):172-7.

6. Gadalla TM. Disability associated with comorbid anxiety disorders in women with chronic physical illness in Ontario, Canada. Women Health. 2008;48(1):1-20.

7. Li Z, Bowerman S, Heber D. Health ramifications of the obesity epidemic. Surg Clin North Am. 2005;85:681-701.

8. Gadalla TM. Association de l'obésité avec les troubles de l'humeur et les troubles anxieux chez l'ensemble de la population adulte. Maladies chroniques au Canada. 2009;30(1):31-40. 
9. Larsson U, Karlsson J, Sullivan M. Impact of overweight and obesity on health-related quality of life - a Swedish population study. Int J Obesity Relat Metab Disord. 2002;26:417-24.

10. Lowry R, Galuska DA, Fulton JE, Wechsler H, Kann L. Weight management goals and practices among U.S. high school students: associations with physical activity, diet, and smoking. J Adolesc Health. 2002;31(2):133-44.

11. Seo DC, Jiang N, Kolbe LJ. Association of smoking with body weight in US high school students, 1999-2005. Am J Health Behav. 2009;33(2):202-12.

12. McCracken M, Jiles R, Blanck HM. Health behaviors of the young adult U.S. population: behavioral risk factor surveillance system, 2003. Prev Chronic Dis. 2007;4(2):A25.

13. Tremblay MS, Willms JD. Secular trends in the body mass index of Canadian children. CMAJ. 2000;163(11):1429-33.

14. Pisetsky EM, Chao YM, Dierker LC, May AM, Striegel-Moore RH. Disordered eating and substance use in high school students: Results from the Youth Risk Behavior Surveillance System. Int $\mathrm{J}$ Eat Disord. 2008;41(5):464-70.

15. Poulin C, Van Til L, Wilbur B, Clarke B, MacDonald CA, Barcelo A, et collab. Alcohol and other drug use among adolescent students in the Atlantic provinces. Can J Public Health. 1999;90:27-9.

16. Hotton T, Haans D. Consommation d'alcool et de drogues au début de l'adolescence. Rapports sur la santé. 2004;15(3):9-19.

17. Tremblay MS, Willms JD. Is the Canadian childhood obesity epidemic related to physical inactivity? Int $\mathrm{J}$ Obesity Relat Metab Disord. 2003;27:1100-5.

18. Babey SH, Hastert TA, Wolstein J, Diamant AL. Income disparities in obesity trends among California adolescents. Am J Public Health. 2010;100(11):2149-55.

19. Chao YM, Pisetsky EM, Dierker LC, Dohm F, Rosselli F, May AM, et al. Ethnic differences in weight control practices among U.S. adolescents from 1995 to 2005. Int J Eat Disord. 2008;41(2):124-33.
20. Garry JP, Morrissey SL, Whetstone LM. Substance use and weight loss tactics among middle school youth. Int $\mathrm{J}$ Eat Disord. 2003;33(1):55-63.

21. Ho CH, Kingree JB, Thompson MP. Associations between juvenile delinquency and weight-related variables: analyses from a national sample of high school students. Int J Eat Disord. 2006:39(6):477-83.

22. Allison KR, Adlaf EM, Ialomiteanu A, Rehm J. Predictors of health risk behaviours among young adults: analysis of the National Population Health Survey. Can J Public Health. 1999;90(2):85-89.

23. Kestila L, Rahkonen O, Martelin T, Lahti-Koski M, Koskinen S. Do childhood social circumstances affect overweight and obesity in early adulthood? Scand J Public Health. 2009;37:206-19.

24. Enquête sur la santé dans les collectivités canadiennes : fichier de microdonnées à grande diffusion. 2007-2008 (cycle 4.1). Ottawa (Ont.) : Statistique Canada; 31 août 2009.

25. Enquête sur la santé dans les collectivités canadiennes : fichier de microdonnées à grande diffusion. 2000-2001 (cycle 1.1) [Internet]. Ottawa (Ont.) : Statistique Canada; 2003 [Consultation le 28 janv. 2003].

26. Cole TJ, Bellizzi MC, Flegal KM, Dietz WH. Establishing a standard definition for child overweight and obesity worldwide: international survey. BMJ. 2000;320:1240-3. 